\title{
Digital systems supporting cognition and exploratory learning in twenty-first century: guest editorial
}

\author{
J. Michael Spector ${ }^{1} \cdot$ Dirk Ifenthaler ${ }^{2}$ • \\ Demetrios G. Sampson ${ }^{3}$
}

\begin{abstract}
Digital systems and digital technologies are globally investigated for their potential to transform learning, teaching and assessment towards offering unique learning experiences to the twenty-first century learners. This Special Issue on Digital systems supporting cognition and exploratory learning in twenty-first century aims to contribute to the global dialogue between the educational technology and educational psychology research community and the educational practitioners on current issues towards large scale take-up of educational technology.
\end{abstract}

Keywords Digital systems · Cognition - Collaborative problem solving · Exploratory learning · Learning communities · Remote labs · Self-regulated learning $\cdot$ Technology integration

\section{Introduction}

The Cognition and Exploratory Learning in the Digital Age (CELDA; http://www. celda-conf.org) conference is a unique international research conference that brings together educational technology and educational psychology researchers as well as instructional designers and other educational practitioners for the purpose of

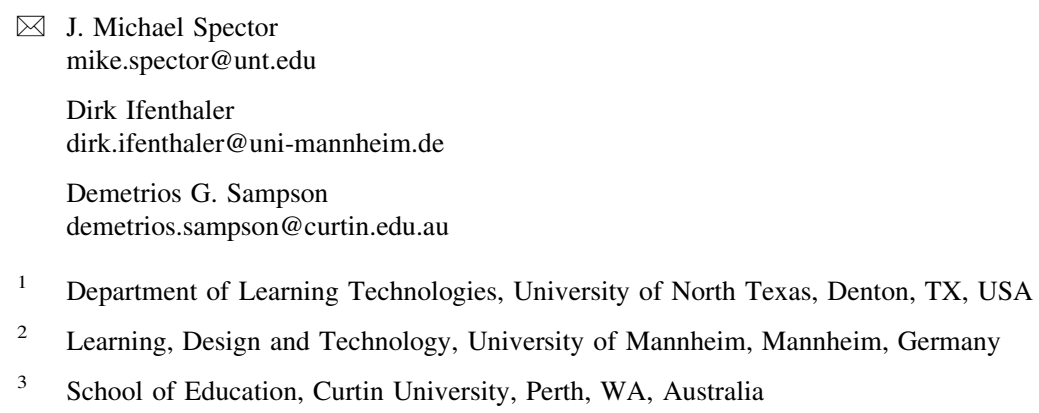


fostering and promoting ongoing dialogue between these academic and professional communities. CELDA is sponsored by the International Association for the Development of the Internet Society (IADIS; see http://www.iadisportal.org/). The disparate nature of these educational technology research and professional groups worldwide results in sporadic and fragmented efforts that limit the transformative potential of digital technologies in the areas of learning, teaching and assessment. This is occurring when there is a global need for improved learning and the tools to support such improvements are widely available. To this end, CELDA has created, since it was initiated in 2004, a community that actively contributes to this dialogue and has contributed to outcomes in the form of special issues published in academic journals and edited books that inform and influence academic and professional practice (Ifenthaler et al. 2011; Isaías et al. 2012; Sampson et al. 2013, 2014; Spector et al. 2010, in press).

This special issue and a forthcoming edited book based on papers presented in CELDA 2015 are the most recent outcomes of this well-established process (Ifenthaler et al. 2012a, b, 2014; Kinshuk et al. 2007, 2008, Kinshuk et al. 2009a, b, 2010; Kinshuk and Sampson 2006). It is created from the extended versions of the best papers around a core theme from the 2015 International Conference on Cognition and Exploratory Learning in the Digital Age (CELDA; see http://www.iadisportal.org/celda-2015-proceedings) that was held in Maynooth, Greater Dublin, Ireland in October 2015, hosted by Maynooth University. Each contribution reports an original research work in the theme of this special issueDigital systems supporting cognition and exploratory learning in twenty-first century.

What follows are six papers that have been expanded from the CELDA 2015 papers: (1) "Extending the will, skill, tool model of technology integration: adding pedagogy as a new model construct" by Gerald Knezek and Rhonda Christensen, (2) "How does self-regulated learning relate to active procrastination and other learning behaviors?" by Masanori Yamada, Yoshiko Goda, Takeshi Matsuda, Hiroshi Kato, and Hiroyuki Miyazawa, (3) "The relationship between collaboration, multi-tasking and problem solving performance in shared virtual spaces" by Lin Lin, Leila A. Mills, and Dirk Ifenthaler, (4) "Comparing learner community behaviour in multiple presentations of a massive open online course" by Silvia Elena Gallagher and Timothy Savage, (5) "Postgraduate Students' Level of Dependence on Supervisors in Coping with Academic Matters and Using Digital Tools" by Gurnam Kaur Sidhu, Sanjit Kaur, Peck Choo Lim, Yuen Fook Chan, and Leele Susana Jamian, and (6) "A study on the use of a metadata schema for characterizing school education STEM lesson plans by STEM teachers" by Panagiotis Zervas, Eleftheria Tsourlidaki, Yiwei Cao, Sofoklis Sotiriou, Demetrios Sampson, and Nils Faltin. The order is based loosely on a general to more specific sequence, with each of the papers focusing issues pertinent to planning and supporting exploratory learning in the digital age.

The special issue starts with a paper by Gerald Knezek and Rhonda Christensen that presents an expanded framework to support planning and technology integration in the digital age. Their paper includes an analysis of three sets of data to support the addition of pedagogical style to the will, skill and tools model 
previously developed and published. This addition has strong implications for teacher preparation and professional development in the context of integrating technology effectively in school environments.

Masanori Yamada and colleagues present an empirical study involving 179 firstyear university students engaged in a blended learning environment. The purpose was to investigate the relationships between self-regulated learning, procrastination and learning behaviors. Data collected included pre- and past-course surveys, log data, and student artifacts (e.g., short papers and reports). Their findings indicate that self-regulation and procrastination are significant factors in understanding learning behaviour. These results have implications for planning motivational and other learning support interventions for different students depending on their levels of self-regulation and tendency to procrastinate.

Lin Lin, Leila Mills, and Dirk Ifenthaler present an empirical study aimed at understanding collaborative problem solving by university students in a shared virtual space. Specific hypotheses involved (a) the relationships between multitasking, collaboration and problem solving, and (b) the extent to which collaboration was positively correlated with problem-solving performance. While there is research to suggest that multi-tasking generally degrades task performance, this study suggests that collaborative problem solving and multi-tasking had a positive effect on task performance. Moreover, collaboration in general tended to improve performance.

Silvia Gallagher and Timothy Savage contribute to research on massive open online courses (MOOCs) by investigating learner communication, expectations and learning behaviors in two iterations of a Futurelearn History MOOC. Retention in the course, learning behaviors, and expectations were analysed taking into account prior online experiences and a number of demographic variables. The MOOC was not changed between the two offerings so that a comparative analysis could be performed. As has been reported for many other MOOCs, many learners reported that they dropped out because they could not keep up with the pace of the course. With regard to learner behaviors, it seems that those from Western cultures are more likely to be actively engaged and make comments than those from Eastern cultures. Such findings as these can help those who plan and support MOOCs create environments that are more likely to engage a variety of learners and reduce the high MOOC drop-out rates.

Gurnam Kaur Sidhu and colleagues report an investigation of 132 postgraduate university students in Malaysia with regard to their perceptions about dependence on supervisors and major professors in relation to their use of digital tools, academic performance and motivation. They found that these students were generally more dependent on their supervisors with regard to academic matters but not really so dependent on their supervisors with regard to the use of digital tools and technologies. While the progress of these students was good when developing and implementing technology-based interventions, when it came to data collection, analysing findings and writing their progress slowed considerably as they became much more dependent on their supervisors. This further suggests that these students may be lacking sufficient autonomy for lifelong academic learning. Implications for addressing this situation in Malaysia and perhaps other cultures are then suggested. 
Concluding this special issue is a paper by Panagiotis Zervas and his colleagues that presents results from a European initiative, namely, the Global Online Science Labs for Inquiry Learning at School (Go-Lab). The paper focuses on the GoLAB technical infrastructure for providing access to STEM (science, technology, engineering and mathematics) lesson plans in association with online laboratories and a study on its use by real teachers. This infrastructure involves a web-based repository where those lesson plans can be placed, and a systematic way to tag those lesson plans to facilitate retrieval and re-use. Then, the authors analyse the metadata records of lesson plans produced by more than a hundred school teachers to better understand their tagging behavior.

Overall, these six selected papers in this special issue demonstrate multiple perspectives and approaches with implications for the transformative potential of digital technologies and digital systems in planning and supporting learning, performance and instruction. We are hopeful that this special issue contributes in a substantive way to the public discourse and research with regard to advanced technologies and innovative approaches to learning and teaching.

Acknowledgments The guest editors of this special issue are grateful for all support received from Professor M. J. Bishop, Editor-in-Chief of the Journal of Computing in Higher Education and the keynote speaker at CELDA 2015.

\section{Compliance with ethical standards}

Conflict of interest The authors declare that they have no conflict of interest.

\section{References}

Ifenthaler, D., Isaías, P., Kinshuk, Sampson, D. G., \& Spector, J. M. (2012a). Technology supported cognition and exploratory learning: Guest editorial. Educational Technology \& Society, 15(1). Retrieved from http://www.ifets.info/journals/15_1/1.pdf.

Ifenthaler, D., Isaías, P., Sampson, D. G., \& Spector, J. M. (2012b). Cognition and exploratory learning supported by technology: Guest editorial. Technology, Instruction, Cognition and Learning, 9, 59-61.

Ifenthaler, D., Kinshuk, Isaías, P., Sampson, D. G., \& Spector, J. M. (Eds.). (2011). Multiple perspectives on problem solving and learning in the digital age. New York: Springer.

Ifenthaler, D., Spector, J. M., Sampson, D. G., \& Isaias, P. (2014). Cognition and exploratory learning in digital age: Guest editorial. Computers in Human Behavior, 32, 290-291.

Isaías, P., Ifenthaler, D., Kinshuk, Sampson, D. G., \& Spector, J. M. (Eds.). (2012). Towards learning and instruction in Web 3.0. Advances in cognitive and educational psychology. New York: Springer.

Kinshuk, Ifenthaler, D., Spector, J. M., Sampson, D. G., \& Isaías, P. (2010). Cognition and learning in the age of digital technologies and social networking. Guest editorial. Journal of Research on Technology in Education, 43(4), 101-102.

Kinshuk, \& Sampson, D. G. (2006). Cognition and exploratory learning in the digital age: Guest editorial. Innovations in Education and Teaching International, 43(2), 105-108.

Kinshuk, Sampson, D. G., Isaías, P., Spector, J. M., \& Schrum, L. (2007). A critical view of technologyenhanced learning and instruction in the digital age: Guest editorial. Journal of Research on Technology in Education, 40(1), 2-3.

Kinshuk, Sampson, D. G., Spector, J. M., Isaías, P., \& Ifenthaler, D. (2009). Cognition and learning technology. Special issue editorial. Educational Technology Research \& Development Journal, 57(6), 721-723. 
Kinshuk, Spector, J. M., \& Sampson, D. G. (2008). Cognition and exploratory technology-enhanced learning: Guest editorial. Computers in Human Behavior, 24(2), 119-121.

Kinshuk, Spector, J. M., Sampson, D. G. \& Isaías, P. (2009b). Cognition and exploratory learning in digital age. Guest editorial. Technology, Instruction, Cognition and Learning, 6(4). Retrieved from http://www.oldcitypublishing.com/TICL/TICLcontents/TICLv6n4contents.html.

Sampson, D. G., Ifenthaler, D., Spector, J. M., \& Isaías, P. (Eds.). (2014). Digital systems for open access to formal and informal learning. New York: Springer.

Sampson, D. G., Isaías, P., Ifenthaler, D., \& Spector, J. M. (Eds.). (2013). Ubiquitous and mobile learning in the digital age. New York: Springer.

Spector, J. M., Ifenthaler, D., Isaías, P., Kinshuk, \& Sampson, D. G. (Eds.). (2010). Learning and instruction in the digital age: Making a difference through cognitive approaches, technologyfacilitated collaboration and assessment, and personalized communications. New York: Springer.

Spector, J. M., Ifenthaler, D., Sampson, D. G., \& Isaías, P. (2016). Competencies in teaching, learning and educational leadership in the digital age. New York: Springer.

J. Michael Spector is a Professor and Former Chair of Learning Technologies at the University of North Texas and a Visiting Professor at the University of Hong Kong. He was previously Professor of Educational Psychology and Instructional Technology, Doctoral Program Coordinator for the Learning, Design, and Technology Program, and a Research Scientist at the Learning and Performance Support Laboratory at the University of Georgia. Previously, he was Associate Director of the Learning Systems Institute, Professor of Instructional Systems, and Principal Investigator for the International Center for Learning, Education and Performance Systems at Florida State University. He served as Chair of Instructional Design, Development and Evaluation at Syracuse University and Director of the Educational Information Science and Technology Research Program at the University of Bergen. He earned a Ph.D. in Philosophy from The University of Texas at Austin. His research focuses on intelligent support for instructional design, assessing learning in complex domains, and technology integration in education. Dr. Spector served on the International Board of Standards for Training, Performance and Instruction (ibstpi) as Executive Vice President; he is a Past President of the Association for Educational and Communications Technology as well as a Past Chair of the Technology, Instruction, Cognition and Learning Special Interest Group of AERA; he is also an active member of AERA's Instructional Technology and Learning and Technology SIGs. He is editor of Educational Technology Research \& Development and serves on numerous other editorial boards. He edited the third and fourth editions of the Handbook of Research on Educational Communications and Technology, as well as the Encyclopedia of Educational Technology, and has more than 150 publications to his credit.

Dirk Ifenthaler is Professor and Chair of Learning, Design and Technology at University of Mannheim, Germany, Adjunct Professor at Deakin University, Australia and Affiliate Research Scholar at the University of Oklahoma, USA. His previous roles include Professor and Director, Centre for Research in Digital Learning at Deakin University, Australia, and Professor for Applied Teaching and Learning Research at the University of Potsdam, Germany. He was a 2012 Fulbright Scholar-in-Residence at the University of Oklahoma, USA. Dirk Ifenthaler's research focuses on the intersection of cognitive psychology, educational technology, learning science, data analytics, and computer science. His research outcomes include numerous co-authored books, book series, book chapters, journal articles, and international conference papers, as well as successful grant funding in Australia, Germany, and USA-see Dirk's website for a full list of scholarly outcomes at www.ifenthaler.info. He is the Editor-in-Chief of the Springer journal Technology, Knowledge and Learning (www.springer.com/10758).

Demetrios G. Sampson is a Professor of Learning Technologies at the School of Education, Curtin University, Perth, Australia. He is also affiliated with the Information Technologies Institute, Centre of Research and Technology-Hellas Greece, European Union, as an Adjunct Research Fellow and with the the School of Computing and Information Systems, Athabasca University, Canada, as an Adjunct Professor. Previously, he has been a Professor of Digital Systems for Learning and Education at the Department of Digital Systems, University of Piraeus, Greece (2003-2015) and a Senior Researcher at the Informatics and Telematics Institute, Centre of Research and Technology-Hellas Greece (2000-2003). $\mathrm{He}$ is the Founder and Director of the Research Program on Advanced Digital Systems and Services for 
Education and Learning (ASK) since 1999. His main scientific interests are in the area of Learning Technologies and Technology Enhanced Teaching and Learning. He has supervised 82 research students to successful completion. He is the co-author of 380 publications in scientific books, journals and conferences with more than 3520 citations and h-index 26 as listed in Scholar Google (June 2016). He has received 8 times Best Paper Award in International Conferences on Learning Technologies. He has been a Keynote/Invited Speaker in 70 International/National Conferences. He has been project director, principle investigator and/or consultant in $66 \mathrm{R} \& \mathrm{D}$ projects with external funding at the range of 15 Million $€$ (1991-2016). He is the Editor-in-Chief of the Educational Technology and Society Journal (5-year impact factor 1.376). He has served or serves as Member of the Steering Committee of the IEEE Transactions on Learning Technologies, Member of the Advisory Board of the Journal of King Saud UniversityComputer and Information Sciences and the International Journal of Digital Literacy and Digital Competence, Member of the Editorial Board of 14 International/National Journals and a Guest Editor in 32 Special Issues of International Journals. He has served or serves in various leadership roles in 62 International Conferences and at the Program Committee of 436 International/National Conferences. He is a Senior Member of IEEE and Golden Core Member of IEEE Computer Society and the elected Chair of the IEEE Computer Society Technical Committee on Learning Technologies since June 2016. He is the recipient of the IEEE Computer Society Distinguished Service Award (July 2012). He has received a Diploma in Electrical Engineering from the Democritus University of Thrace, Greece in 1989 and a Ph.D. in Electronic Systems Engineering from the University of Essex, UK in 1995. 